\title{
MicroRNA-466c-3p exerts protective effect on neuronal apoptosis and improves functional recovery post spinal cord injury via mitochondrial apoptotic pathway
}

\author{
Yan An, Jianing Li, Qiang Yuan * (1) and Mingxing Fan
}

\begin{abstract}
Spinal cord injury (SCl) is involved with abnormal expression of miRNAs (miRs) which are responsible for some $\|^{\text {ry }}$ injury responses which include apoptosis, inflammation and oxidative stress. Mechanisms involving miRs induced apoptosis still needs to be investigated. In the present work we developed a rat model of SCl, followed by microarray analysis for expression of miRs at various time points after $\mathrm{SCl}$. The locomotor activity was assessed by Basso, Beattie and Bresnahan score, lesion volume was analyzed by cresyl violet staining and TUNEL staining for extent of apoptosis at various time points of post $\mathrm{SCl}$. Numbers of miRs were altered after 2 weeks of SCl among which miR-466c-3p was the most significantly down-regulated. Transfection with miR-466c-3p mimics caused overexpression of miR-466c-3p, also improvement in functional recovery, decrease in apoptosis of neuronal cells and lesion size was observed in SCl rats. The Luciferase assay suggested that miR-466c-3p suppressed the expression of $\mathrm{BCl}-2$ (apoptosis regulator). It was also evidenced that upon restoring the levels of $\mathrm{BCl}-2$ with the help of pc-DNA3-BCl-2 halted the attenuating action of miR-466c-3p in hydrogen peroxide exposed N9 microglia cells. The findings suggested that miR-466c-3p may inhibit mitochondrial apoptotic pathway via blocking BCl-2 and cleaved capase-9/-3in rats after SCl. Altogether, the results suggested that miR-466c-3p may exert attenuating effect on functional recovery and inhibit the apoptosis of neuronal cells via halting the mitochondrial apoptosis cascade in $\mathrm{SCl}$ rats indicating that miR-466c-3p can be attractive therapeutic candidate in treating $\mathrm{SCl}$.
\end{abstract}

Keywords: miR-466c-3p, Spinal cord injury, Bcl-2, Apoptosis

\section{Introduction}

Spinal cord injury (SCI) is a common injury of spinal cord leading to permanent disability which includes paralysis, loss of sensitivity, control and movements in the lower part of body below the affected region (Chen et al. 2016). The global rate of SCI is alarming; treatment for SCI is biggest challenge with the neurologists worldwide (Thuret et al. 2006). Apoptosis of neuronal cells is the major hurdle in SCI as the neurons play an important

*Correspondence: ay_jstspine@vip.163.com

Department of Spine Surgery, Beijing Jishuitan Hospital, Beijing 100035, China role in functional as well as physical deficits (Blight 2002; Rabchevsky et al. 2011), hence developing novel therapeutic approaches targeting and halting apoptosis is of outmost importance in managing SCI.

Apoptosis is a phenomenon in cells also called as programmed cell death; it is an important process which affects the neuronal tissue damage after SCI (Kawabata et al. 2010). However earlier it has been reported that both mitochondrial pathway and death receptor cause apoptosis (Li et al. 2000). Previously it has been evidenced that Bcl-2 members arbitrate apoptosis signals by involving anti-apoptosis proteins like B cell lymphoma and proteins such as Bax and Bcl-2 which are agonist 
of cell death in the mitochondrial cascade (Reuter et al. 2008). The degeneration of mitochondrial membrane potential is the main process of mitochondrial apoptotic pathway which leads to transfer of cytochrome-c to the cytosol from the mitochondria (Balaban et al. 2005). In addition to this, cytochrome-c along with deoxy ATP and apoptosis protease triggering factor- 1 converts cleaved pro-caspase- 9 into active-caspase- 9 in the cytosol ( $\mathrm{Li}$ et al. 2004). Further, this active-caspase- 9 causes cleavage of caspase-3/-6/-7 (Nicholson and Thornberry 1997). Hence the release of cytochrome-c is an important step for the activation of pro-caspase-9 in apoptosis of cells.

miRNAs (miRs) are identified to be small noncoding RNAs having single strand measuring about 21-24 nucleotides. These micro-molecules are responsible for modulating the expression of target genes at post-transcriptional level by causing degradation of RNA or suppressing the translation (Croce 2009; Bartel 2009; Liu et al. 2009). Numerous miRs have been discovered to be involved in regulating the development of neuronal cells in mammalian brain and spinal cord (Bak et al. 2008; Kosik 2006). In addition to this some miRs have also been identified to play potential role in development of neurons and are believed to be important mediators contributing in cell differentiation (Kosik 2006). Studies earlier have showed that SCI may cause aberrant expression of miRs, which is feature of many responses of secondary injuries such as apoptosis, inflammation and oxidative stress (Liu et al. 2010; Wang et al. 2017). A study recently has suggested that miRs could regulate apoptosis via mitochondrial apoptosis cascade in various disorders (Yune et al. 2007).

In the present work, we created an SCI rat model followed by microarray analysis for determining the expression profiles of miRs in the injured spinal cord tissue. In addition to this, the role of miR-466c-3p in spinal injury mediated apoptosis was evaluated followed by the mechanism involved in the mitochondrial apoptosis cascade was investigated.

\section{Materials and methods \\ Cell culture}

For the present work we selected murine N9 microglia cell line which was procured from the Institute of Neuroscience, IVth Military Medical University, China. The use of cell lines received approval from the ethical board of IVth Military Medical University, China. The cell lines were cultured and stored in Dulbecco's modified Eagle's medium/F12 (DMEM) media supplemented with fetal bovine serum (10\%) (FBS), streptomycin and penicillin $(100 \mathrm{U} / \mathrm{ml})$ maintained at $37{ }^{\circ} \mathrm{C}$ under humid conditions with $5 \% \mathrm{CO}_{2}$ in an incubator.

\section{Cell treatments}

The selected N9 microglia cell lines were exposed to various concentrations of hydrogen peroxide $(30 \% \mathrm{w} / \mathrm{w})$ (Sigma-Aldrich USA) for $10 \mathrm{~h}$ for inducing cell injury. The concentrations of hydrogen peroxide were 50 to $400 \mu \mathrm{M}$ in phosphate buffered saline solution.

\section{Experimental animals and spinal cord injury model}

For creating SCI animal model, we selected adult Sprague-Dawley rats (female) aging about 6 weeks and weighing between 210 and $240 \mathrm{~g}$. The animals were obtained from the Beijing Jishuitan Hospital, Beijin, China. The animal study protocols were approved by animal ethical review board of Beijing Jishuitan Hospital, Beijin, China. The rats were housed in polypropylene cages under pathogen free conditions with relative humidity ranging between 55 and $65 \%$ and temperature $23 \pm 1{ }^{\circ} \mathrm{C}$ following $12 \mathrm{~h}$ dark-light cycle. The rats were given free access to water and food (pellet diet). The rats were distributed into four groups viz. the SCI group (control), Sham operated group, miR-466c-3p mimics and antago-miR-466c-3p group ( $\mathrm{NC}$ control), and all the groups had 8 rats. For inducing SCI, the rats were subjected to weight drop injury to the spinal injury at the T10 spinal disc using an impactor. For sham operated group the rats were subjected to T10 laminectomy without performing the weight drop injury. In the miR$466 c-3 p$ mimics treated group the rats were subjected to $\mathrm{SCI}$ and the received intrathecal dose of $1 \mu \mathrm{l} / \mathrm{h} \mathrm{miR-}$ $466 \mathrm{c}-3 \mathrm{p}$ mimics $(20 \mathrm{nmol} / \mathrm{ml})$ for next 3 days. For the negative control $(\mathrm{NC})$ rats the $\mathrm{SCI}$ induced rats were given treatment of miR-466c-3p antagomir via intrathecal route $(1 \mu \mathrm{l} / \mathrm{h}, 20 \mathrm{nmol} / \mathrm{ml})$.

The rat model of spinal cord injury was created by submitting the rats to pentobarbital anesthesia followed by laminectomy of the T9-T10 level for revealing the spinal cord tissue, care was taken that no damage was done to the dura. The spine was stabilized by clamping the T8 and $\mathrm{T} 11$ processes, after this the dorsal surface of spine was submitted to weight drop injury using an impactor as reported earlier (Basso et al. 1995). The sham operated rats were submitted to laminectomy without performing the weight drop process for injury.

\section{miRNA microarray analysis}

The spinal tissue of SCI injured rats were assessed for miRNA expression by miR micro array analysis, at least 2 rats from each group were subjected to Pentobarbital anesthesia and sacrificed after 2 weeks of SCI. The rats were operated and a $10 \mathrm{~mm}$ piece of spinal cord which also included the epicenter of injury was dissected and freezed in liquid nitrogen. The spinal tissue was 
processed with TRIzol reagent and total RNA was isolated and purified with the help of RNeasy kit (Qiagen, Germeny) following the supplied instructions. The samples were analyzed for RNA content using spectrophotometer (ThermoFisher USA), the isolated miRs were then hybridized on a LNA array. The resultant slides were scanned using an Axon Genepix microarray scanner. The images were analyzed using GenePix software version 6 . The miRs showing intensities greater than or equal to 50 in all the samples were employed for calculating the normalization factor. The miRs were studied by the volcano plot filtering method.

\section{Isolation of RNA and qRT-PCR}

Isolation of RNA was done from the spinal cord tissue isolated from the SCI rats, care was taken that the tissue had the damaged portion. TRIzol reagent (ThemoFisher USA) was used for isolating RNA following the supplied instructions. The reverse transcription was done using the Taqman RT kit (ThermoFisher USA) on a Real Time PCR system (ThermoFisher USA). The conditions maintained for thermocycling were temperature of $50{ }^{\circ} \mathrm{C}$ for first $2 \mathrm{~min}$ and then temperature of $95^{\circ} \mathrm{C}$ for next $10 \mathrm{~min}$, and then forty cycles of $15 \mathrm{~s}$ at $95^{\circ} \mathrm{C}$ and next $10 \mathrm{~min}$ at $60{ }^{\circ} \mathrm{C}$. The primer sequences which were utilized for the study were: miR-466c-3p, 3'-CACACAUACACACGU ACAUAU -5' (Forward) and 5'-AUCAGCCGGACCGCG CACCTT CACGC-3' (Reverse); Bcl-2, 5'-CUAUGC AUCUUGUAACAUGUAUU-3' (forward) and 5'-GGT GAGGACTCCAGCCACAA-3' (reverse), all reactions were performed in triplicate.

\section{Behavioral parameters study}

For assessing the behavioral parameters Basso, Beattie and Bresnahan (BBB) scoring was selected for evaluating the locomotor behavior in rats. The study was performed for an interval of 4 min by two trained and experienced investigators also they were kept blank about the experimental conditions. Open field locomotor test was performed for evaluating the hind limb function and the score was calculated in accordance to BBB scale as published earlier (Basso et al. 1995; Tang et al. 2014).

\section{Evaluation of lesion volume}

For assessing the volume of lesion after SCI or after treating the rats with miR-466c-3p mimics, the rats were subjected to Sodium pentobarbital anesthesia $(50 \mathrm{mg} \mathrm{kg})$ via intraperitoneal route. The rats were submitted to trans-cardiac perfusion with isotonic saline solution $(0.9 \%)$ followed by paraformaldehyde solution (4\%) in $0.1 \mathrm{M}$ Phosphate buffered saline $\mathrm{pH}$ 7.4 for $30 \mathrm{~min}$. About $10 \mathrm{~mm}$ portion of spinal cord bearing injured part was harvested and then fixed in paraformaldehyde solution at $4{ }^{\circ} \mathrm{C}$. For obtaining the sections the tissues were fixed in paraffin to form blocks. The sections were obtained using a rotary microtome of $10 \mu \mathrm{m}$ thickness; the sections primarily covered the lesion sites which were then fixed on slides. The sliced tissue sections were stained with Cresyl violet acetate solution $(0.5 \%)$ for $1 \mathrm{~h}$ and the slides were then imaged using Olympus microscope, the damaged area in the sections were quantified with the help of Image-pro software version 6 (Media Cybernetics, USA). The left over portion showing normal tissue area with preserved structure was considered as spared tissue and was reported. The section of tissue with least spared tissue percentage was regarded as the injury epicenter. The range of lesion epicenter was about $400 \mu \mathrm{m}$ caudal and rostral, the area was evaluated through a distance of about $1800 \mu \mathrm{m}$ in the periphery of injured epicenter for the total spared tissue percentage.

\section{TUNEL assay}

For detecting apoptosis, the spinal cord tissue sections previously generated were submitted for TUNEL staining using a TUNEL apoptosis kit (ThermoFisher USA). The tissue sections were dipped into TUNEL reagent for $60 \mathrm{~min}$, after this the nuclei were stained with DAPI solution $(1 \mu \mathrm{g} / \mathrm{ml})$ for $10 \mathrm{~min}$ and then the sections were immersed in Fluoromount media (SigmaAldrich USA). The positive cells were quantified in 10 random field in each slide using florescent microscope (Olympus), the apoptotic cells as well as total cells were counted.

\section{Luciferase reporter assay}

Bioinformatics analysis was performed using TragetScan (http://www.targetscan.org/vert_72/) for identifying the favorable binding site between miR-466c-3p and $\mathrm{Bcl}-2$. For the transfection, the miR-466c-3p mimics and inhibitor were used. The 3'UTR fragment of Bcl-2 wild type as well as mutant were amplified and then cloned in reporter luciferase vector. Further, site directed mutagenase of $3^{\prime} \mathrm{UTR}$ of Bcl-2 was done on the identified binding site of miR-466c-3p with the help of site directed mutagenesis kit. Subsequently, N9 microglia cells were seeded at density of $2 \times 10^{5}$ cells/well in the 24 well plates followed by co-transfection with p-miR-Bcl-2 $3^{\prime}$ UTR or p-miR-Bcl-2-mutant-3'UTR and miR-466c-3p mimics/ inhibitor or negative control with the help of transfecting reagent Lipofectamine (ThermoFisher USA). Renilla luciferase was selected as control for normalizing the cell number after $48 \mathrm{~h}$ of transfection. The luciferase assay was performed with the help of luminescent reporter gene assay (ThermoFisher USA). 


\section{Western blot analysis}

The spinal cord segments having the injured site were harvested and were processed to isolate proteins, the proteins were also isolated from the N9 microglia cells with the help of radio immuno-precipitation buffer (Roche) as discussed in earlier studies (Wang et al. 2017). The resultants' were mixed and centrifuged at $12,000 \mathrm{rpm}$ for $15 \mathrm{~min}$. The supernatants were removed and stored freezed. The expression of proteins was estimated from supernatant using bicinchoninic acid assay also known as BCA assay. About $30 \mu \mathrm{g}$ protein was submitted for electrophoresis on SDS/PAGE (10\%) and then transferred to PVDF membrane. The membranes were blocked with the help of non-fat milk (5\%) for $12 \mathrm{~h}$ at $4{ }^{\circ} \mathrm{C}$ and then incubated with $\mathrm{I}^{\text {ry }}$ antibodies against Bax (1:1000), Bcl-2 (1:1000), Pro-caspase- 3 and cleaved caspase-3 (1:1000), Pro-caspase-9 and cleaved caspase-9 (1:1000) and lastly the loading control Actin (1:1000). All the antibodies were bought from Santa Cruz Biotech. USA. The membranes were incubated for 60 min with anti-mouse IgG horse-radish peroxidaseconjugated secondary antibody. The bands were studied with the help of ImageQuant version 5.2 software.

\section{Immunohistochemical staining}

For removing the blood the spinal tissue was submitted to intracardial perfusion with isotonic solution $(0.9 \%$ sodium chloride) followed by phosphate buffered saline solution of paraformaldehyde solution (4\%) for $30 \mathrm{~min}$. The spinal cord section measuring $10 \mathrm{~mm}$ having the injured site was isolated and fixed in paraffin block and sections were produced of thickness $5 \mu \mathrm{m}$. The sections were deparaffinized using xylene followed by rehydrating them with series of alcoholic dilutions ranging from 100 to $40 \%$. After this the sections were submitted to microwave in $10 \mathrm{mM}$ citrate buffer for $5 \mathrm{~min}$ for epitope unmasking. After this the endogenous peroxidase was inactivated by treating the sections with $\mathrm{H}_{2} \mathrm{O}_{2}$ (3\%) for $10 \mathrm{~min}$. The sections were rinsed with phosphate buffered saline three times and were obstructed with fetal bovine serum $(10 \%)$ and then incubated with anti-cleaved-caspase- 3 antibodies (1:1000). The tissue sections were then again incubated along with anti-mouse IgG horseradish peroxidase IIry antibodies for $30 \mathrm{~min}$. At last the sections were evaluated for immunoreactivity by $3,3^{\prime}$-diaminobenzidine staining for $3 \mathrm{~min}$ and then were cover sliped. The images were recorded using Olympus light microscope and quantification was preformed with the help of Aperio software version 9.

\section{Flow cytometry for apoptosis studies}

About $1 \times 10^{6} \mathrm{~N} 9$ microglia cells were collected and rinsed in phosphate buffered saline for $30 \mathrm{~min}$. Fluorescein isothiocyanate (FITC) and Annexin V staining was done to identify the extent of apoptosis. The cells were rinsed at least thrice with phosphate buffered saline followed by incubation with propidium iodide (PI) $(1 \mu \mathrm{l})$ and Annexin V-FITC $(5 \mu \mathrm{l})$. After staining the cells were analyzed using flow cytometry (ThermoFisher USA).

\section{Determination of Caspase-3 activity}

Colorimetric assay was done for determining caspase-3 activity using a kit following the supplied instructions (SigmaAldrich USA). The N9 cells were isolated by centrifugation at 12,000 rpm for $10 \mathrm{~min}$ and then incubated in lysis buffer for $15 \mathrm{~min}$. Further, the lysates were centrifuged at 12,000 rpm for $15 \mathrm{~min}$, later the protein content was determined by protein estimation kit (SigmaAldrich USA) following the supplied instructions. The isolated lysates were incubated with Ac-DEVD-pNA $(0.2 \mathrm{mM})$ $(10 \mu \mathrm{l})$ reaction buffer for $2 \mathrm{~h}$. The samples were evaluated using microplate reader at $405 \mathrm{~nm}$.

\section{Statistical evaluation}

The statistical studies were performed using Graphpad Software version 6. The results were presented as mean \pm SD. Spearman's rank correlation was done establishing the link between expression of miR-466c-3p and $\mathrm{Bcl}-2$. The variations in the groups were evaluated by one-way ANOVA tukey's post hoc test and the variations in the group were analyzed by student's t-test. $\mathrm{P}<0.05$ was considered as significant.

\section{Results}

$\mathrm{SCl}$ causes aberrant expression of miRNA in rats

In line to evaluate the potential role of miRs in SCI, we created a rat model of SCI followed by microarray analysis for identifying the expression of miRs in the injured spinal cord. We found that expressions of large number of miRs were altered after 2 weeks inducing SCI and among them miR-466c-3p was the most significantly suppressed compared to the sham operated rats (Fig. 1a). In a previous research it was observed that the expression miR-466c-3p decreased significantly with time under spinal injured conditions in rats (Druz et al. 2011). Therefore, qRT-PCR analysis was performed for verifying the expression of miR-466c-3p in spinal cord tissues i.e. on 1st, 7th, 14th and 28th day after inducing spinal cord injury. The outcomes suggested a significant down-regulation in levels of miR-466c-3p in SCI group against the sham operated group observed from the 7 th to the 28 th 


\section{a}

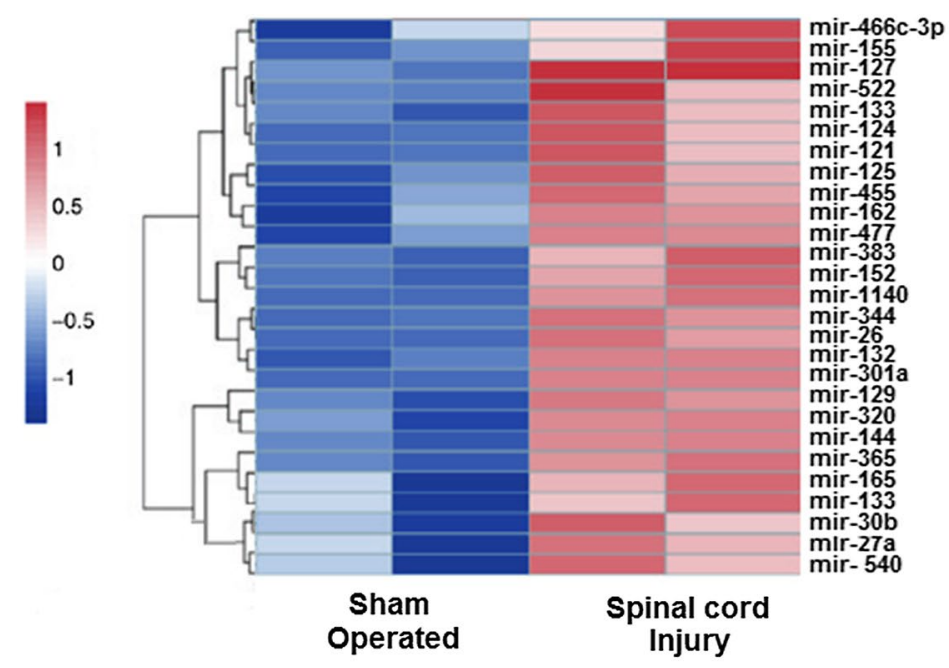

b

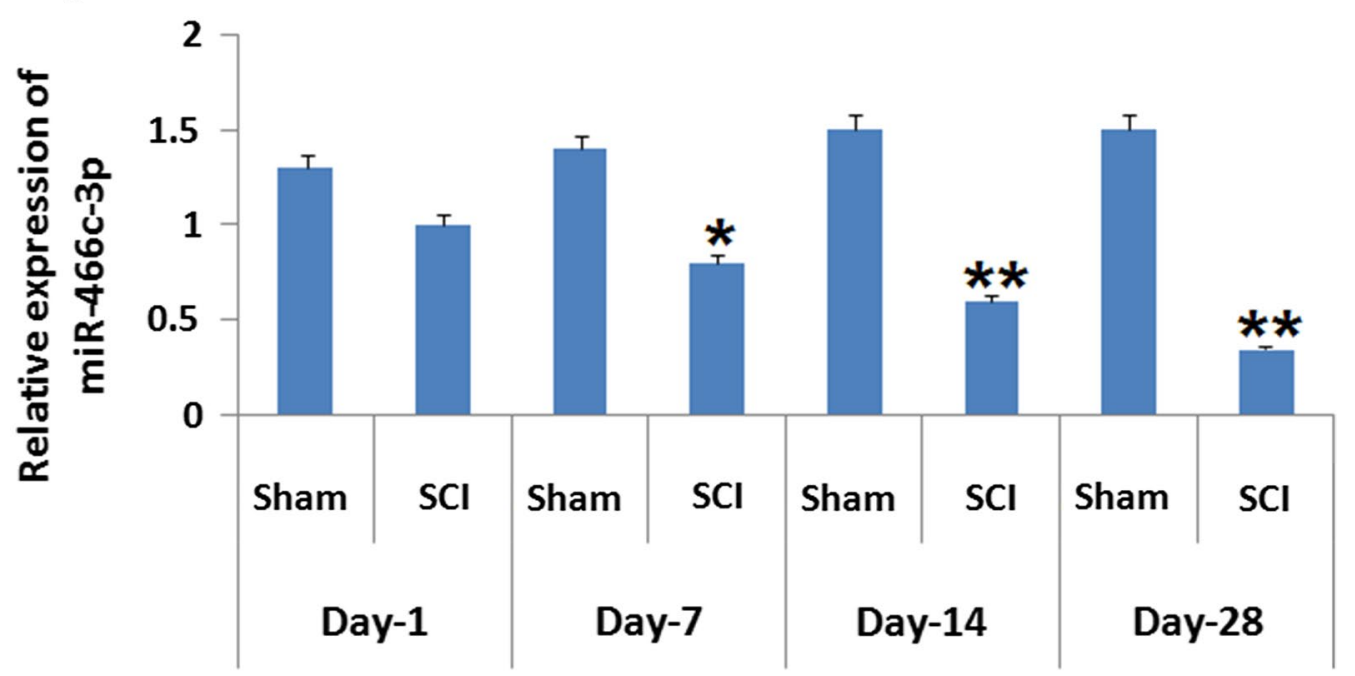

Fig. 1 Expression of miR in rats subjected to spinal cord injury. a Outcomes of heat map analysis showing significant changes in expression of miRs in rats after 14 days subjecting them to spinal cord injury. The blue color shows suppression and red shows over-expression. b Quantitative results of reverse transcription by qRT-PCR for determining the expression of miR-466c-3p in the spinal cord tissues of rats isolated on the 1st, 7th, 14th and the 28th day after inducing spinal cord injury. The results are presented mean $\pm \mathrm{SD}$. ${ }^{*} \mathrm{P}<0.05,{ }^{* *} \mathrm{P}<0.01$ compared to sham operated animals

day $(\mathrm{P}<0.05$; Fig. 1b). The findings indicated that SCI causes ectopic expression of miRs in spinal cord tissues and miR-466c-3p may hold the pathogenesis in animal model of SCI.

\section{Up-regulation of miR-466c-3p attenuated the functional} activity and inhibited apoptosis of neuronal cells in SCI rats To evaluate the function of miR-466c-3p in SCI induced rats, the SCI induced rats were treated with miR-466c-3p mimics given by intrathecal route. After this the spinal cord tissues were harvested and screened for overexpression of miR-466c-3p with the help of qRT-PCR. The results (Fig. 2a) showed that the relative expression of miR-466c-3p was significantly upregulated on the 7 th as well as 28th day compared to the rats treated with miR-466c-3p inhibitor (i.e. agomiR-466c-3p NC) $(\mathrm{P}<0.05)$, also it was found that the expression was highest at the 14th day.

In addition to qRT-PCR analysis for expression of miR$466 c-3 p$, motor function was evaluated by Basso, Beattie, Bresnahan (BBB) scale in the SCI rats after treating them with miR-466c-3p mimics and agomiR-466c-3p NC, the outcomes suggested that upregulation of miR-466c-3p in the spinal cord injury rats treated with miR-466c-3p mimics showed significant improvement in motor function from the 7 th day as compared to spinal cord injury 


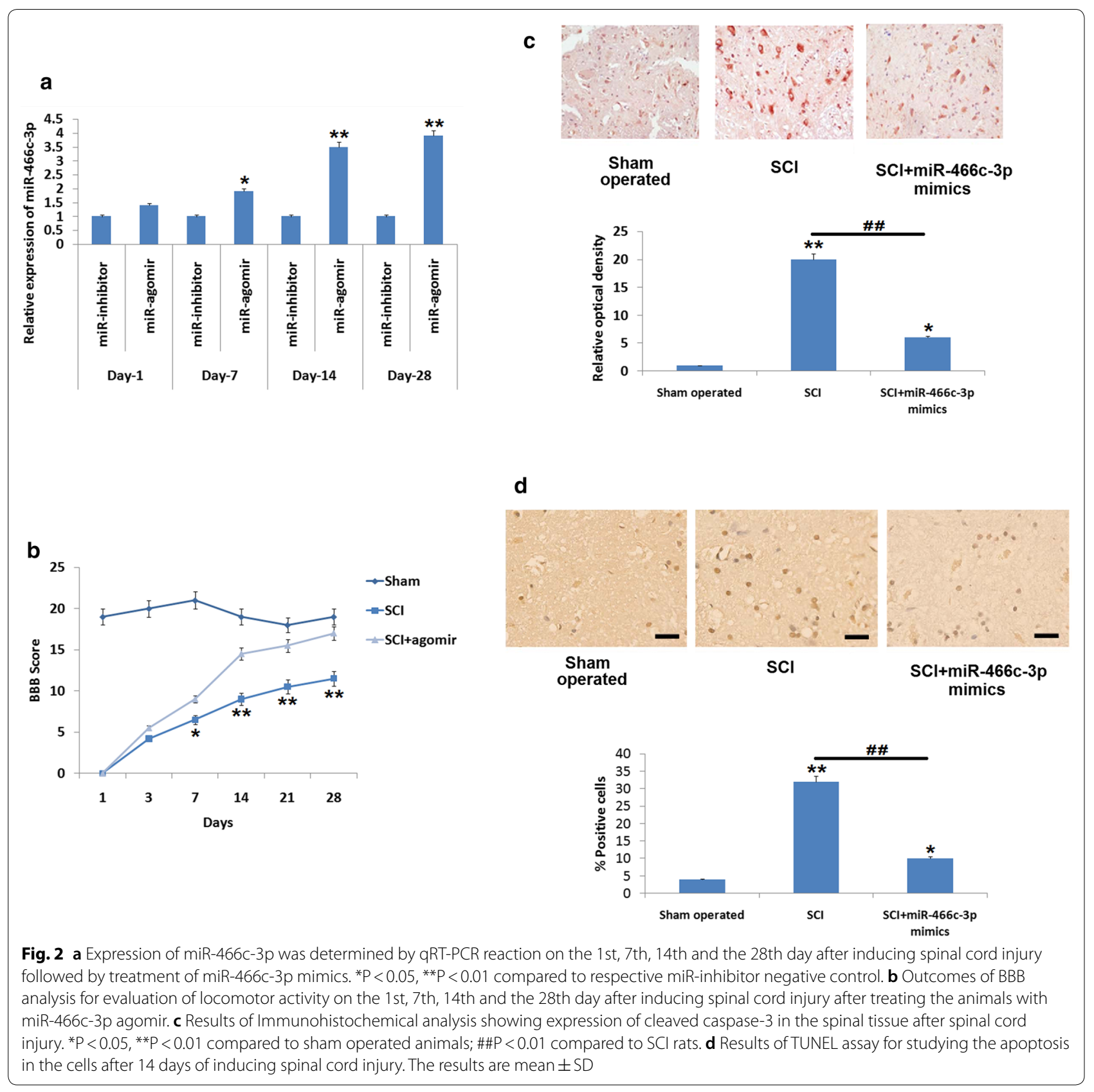

rats (Fig. $2 \mathrm{~b}, \mathrm{P}<0.05$ ). The staining results of Cresyl violet suggested that the normal spared tissue area in sections of miR-466c-3p mimics treated SCI rats increased significantly compared to SCI induced rats, indicating that miR-466c-3p mimics decreases the volume of lesion in the spinal cord tissues after SCI. Further we evaluated using immuno histochemical staining whether miR-466c-3p could regulate the expression of apoptosis related protein i.e. cleaved caspase- 3 in the spinal cord after SCI. As evidenced, SCI caused a substantial overexpression of cleaved-caspase- 3 in the spinal tissues compared to sham operated rats (Fig. 2c), whereas, the treatment of miR-466c-3p mimics caused a significant decrease in levels of cleaved-caspase-3 (Fig. 2c, $\mathrm{P}<0.01$ ). In addition, TUNEL studies were done to assess apoptosis of neuronal cells. The number of TUNEL positive cells increased substantially in rats submitted to SCI against the sham operated rats $(\mathrm{P}<0.01)$, however it was observed that miR-466c-3p mimic suppressed TUNEL positive cells in rats compared to SCI induced 
rats $(\mathrm{P}<0.01 \mathrm{Fig} .2 \mathrm{~d})$. These findings indicated that miR$466 c-3 p$ mimic attenuated motor function, decreases the volume and size of lesion and decrease apoptosis of neuronal cells post $\mathrm{SCI}$.

\section{miR-466c-3p decreases the expression of Bcl-2 via $3^{\prime}-$ UTR in N9 microglia cells}

Studies earlier have confirmed that miR-466 shows protective effect against apoptosis in mammalian cells (Rojo et al. 2014). Hence it was hypothesized that miR-466c-3p may affect apoptosis of neurons in rats subjected to SCI via altering $\mathrm{Bcl}-2$. For predicting potential targets of miR466c-3p, bioinformatics analysis was performed, it was evidenced that $\mathrm{Bcl}-2$ was target gene of miR-466c-3p having potential site located on the $3^{\prime}$-UTR region (Fig. 3a). To corroborate the in silico predicted target, the wild type (WT) and mutant (mut) type Bcl-2 3'-UTR was prepared and was put into the firefly $\mathrm{p}-\mathrm{miR}$ reporter luciferase vector. The pathological factors were studied in N9 microglia cells. The N9 cells were transfected with either miR-466c-3p mimics or inhibitor NC followed by evaluation of luciferase activity. The results suggested that, as compared to inhibitor NC the miR-466c-3p mimics caused significant inhibition of luciferase activity along with wild type $3^{\prime} U T R$, whereas miR-466c-3p inhibitor increased the luciferase activity significantly compared to inhibitor (Fig. 3b, $\mathrm{P}<0.01$ ). In addition to this, miR-466c-3p do not suppressed the luciferase activity in the $3^{\prime} \mathrm{UTR}$ of $\mathrm{Bcl}-2$ containing reporter vector having mutations in the miR-466c-3p binding site (Fig. 3b). To confirm whether, expression of Bcl-2 is negatively regulated by miR-466c-3p, both qRT-PCR and western blot analysis was performed for detecting the mRNA as well as protein levels of $\mathrm{Bcl}-2$. The results demonstrated that, upregulation of miR-466c-3p suppressed the expression of Bcl-2 at both the protein as well as at mRNA levels in N9 microglia cell lines (Fig. 3c, d). Inversely, suppression of miR-466c-3p enhanced the expression of Bcl-2 at mRNA as well as at protein levels (Fig. 3c, d). The outcomes of qRT-PCR were used to evaluate the mRNA levels of Bcl-2 in the spinal cord tissues $(n=8)$. We found that the mRNA levels of Bcl-2 increased significantly in $\mathrm{SCI}$ rats compared to sham operated (Fig. 3e, $\mathrm{P}<0.01$ ). Statistical analysis suggested a significantly negative correlation between expression of miR-466c-3p and Bcl-2 in the spinal cord tissues of SCI rats $(r=-0.901$; Fig. 3f $)$.

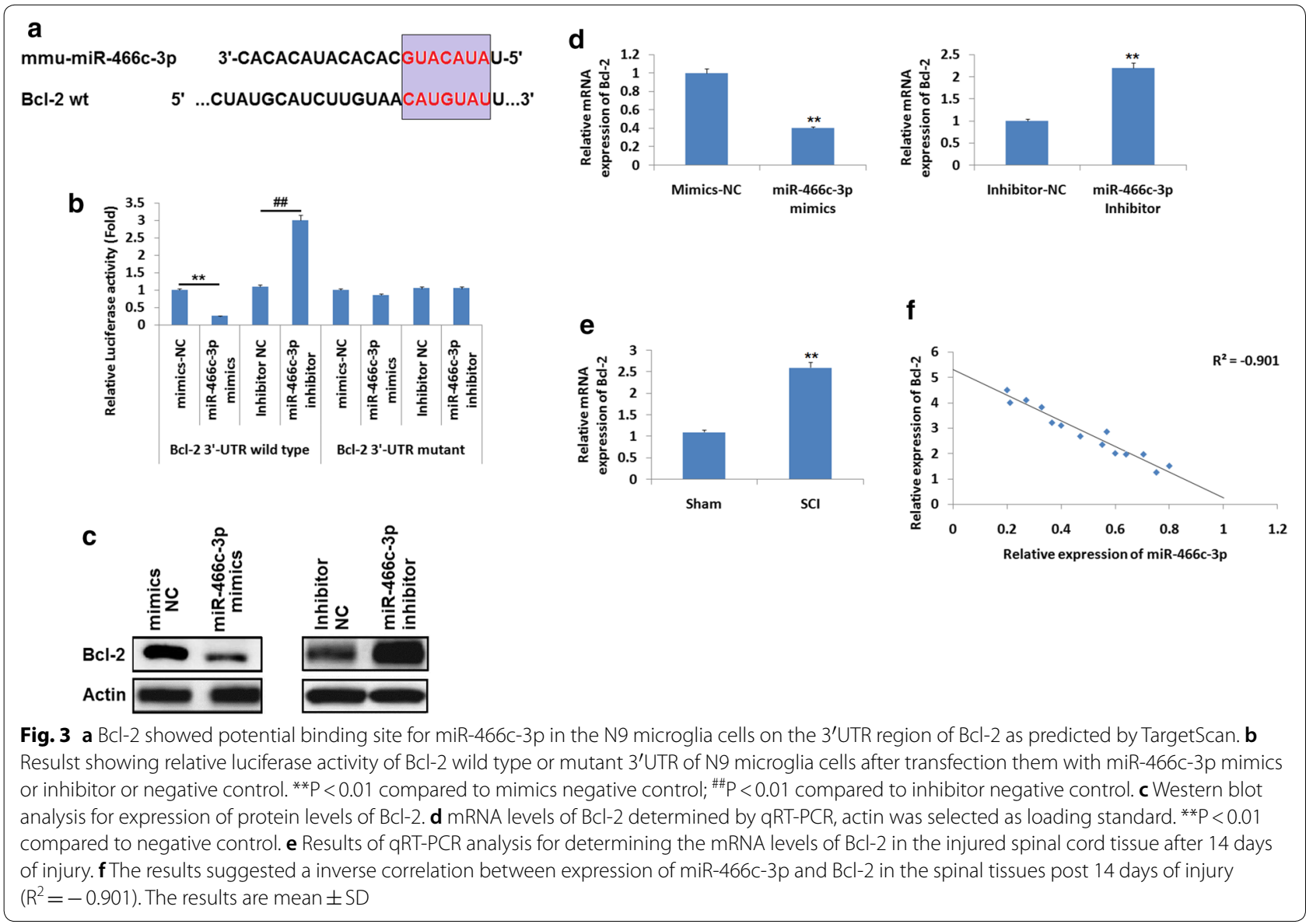


Altogether, the outcomes suggested that miR-466c-3p suppressed the levels of Bcl-2 via targeting the $3^{\prime} \mathrm{UTR}$ of N9 cells directly, indicating Bcl-2 may be the potential target of miR-466c-3p in the injured spinal cord.

\section{Upregulation of $\mathrm{Bcl}-2$ halts the attenuating effect of miR-} $466 \mathrm{c}-3 \mathrm{p}$ on hydrogen peroxide exposed $\mathrm{N} 9$ microglia cells Previous reports have indicated that reactive oxygen species (ROS) play an important role in SCI as ROS can trigger numerous cascade of apoptosis, hydrogen peroxide exposed N9 cells have been used as cellular models of SCI for examining the pathological factors after SCI (He et al. 2000). In the current study, N9 microglia cells were submitted to $\mathrm{H}_{2} \mathrm{O}_{2}$ at varied concentrations $20-320 \mu \mathrm{M}$ for $10 \mathrm{~h}$ and levels of miR$466 c-3 p$ were evaluated with the help of qRT-PCR analysis (Fig. 4a). It was found that exposure of $\mathrm{H}_{2} \mathrm{O}_{2}$ to $\mathrm{N} 9$ cells caused a significant suppression of miR-466c-3p, also the suppression was dose mediated $(\mathrm{P}<0.05)$. In addition to this, qRT-PCR and immunoblot study was
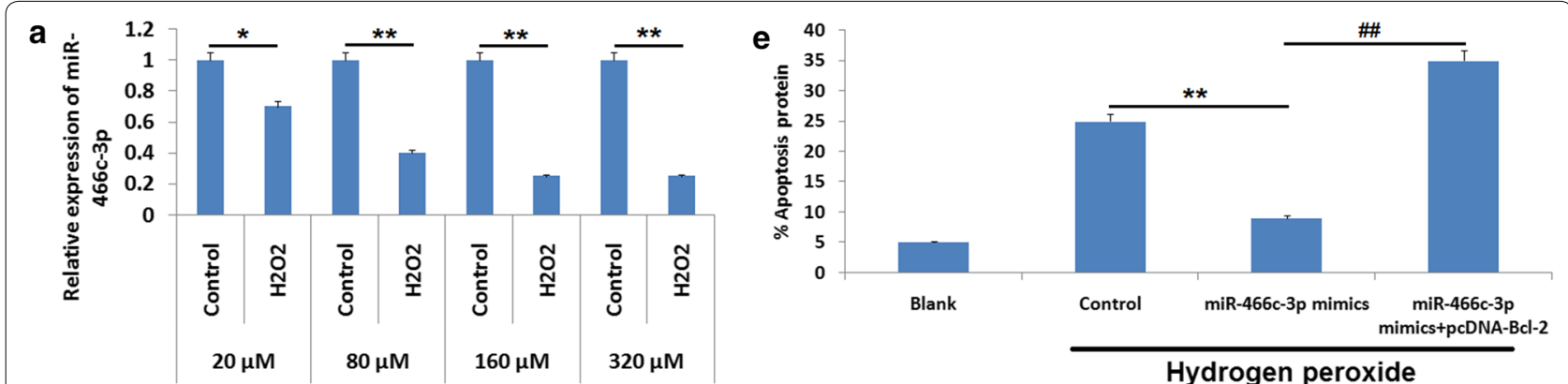

Hydrogen peroxide
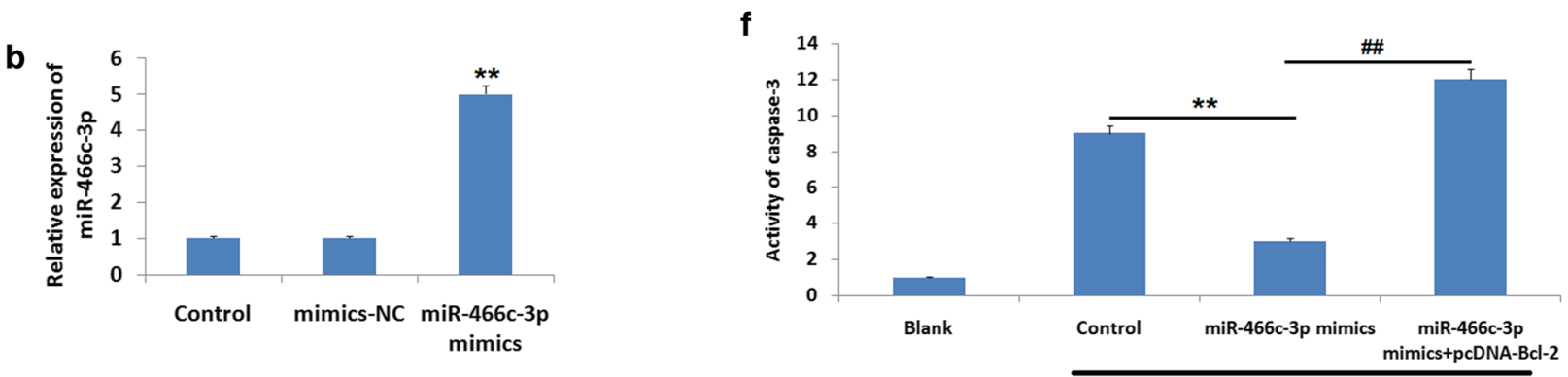

Hydrogen peroxide

C

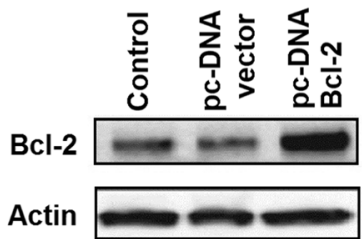

d

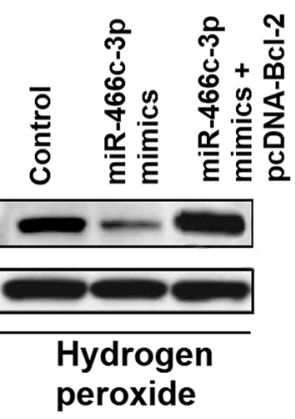

Fig. 4 a The N9 microglia cells were treated with hydrogen peroxide of varied concnetrions for $10 \mathrm{~h}$ followed by measuring the expression by $q R T-P C R .{ }^{*} P<0.05,{ }^{* *} P<0.01$ compared to control. $\mathbf{b}$ qRT-PCR analysis for expression of miR-466c-3p in N9 microglia cells transfected with miR-466c-3p mimics or negative control. ${ }^{*} P<0.05$, ${ }^{*} \mathrm{P}<0.01$ compared to mimice neative control group cells. $\mathbf{c}$ The N9 cells received transfection of pc-DNA-Bcl-2 or pc-DNA-vector followed by evaluation of Bcl-2 by western blot analysis. $\mathbf{d}$ The $\mathrm{N} 9$ cells were transfected with miR-466c-3p mimics or miR-466c-3p mimics along with pc-DNA-Bax and expression of $\mathrm{Bcl}-2$ were estimated by western blot analysis. The cells were treated with hydrogen peroxide followed by transfection with miR-466c-3p mimics or miR-466c-3p mimics and pc-DNA-Bcl-2. e The number of cells under gone apoptosis and $\mathbf{f}$ activity of caspase- 3 were evaluated with the help of flow cytometry and colorimetric analysis respectively. ${ }^{* * P}<0.01$, \#\#P<0.01 compared to control and mimics respectively 
done to assess the upregulation efficacy of miR-466c-3p or Bcl-2. The outcomes suggested that, expression of miR-466c-3p and Bcl-2 was upregulated in N9 cells exposed to pc-DNA-BAx and miR-466c-3p mimics respectively (Fig. 4b, c). Also, it was noticed that upregulation of miR-466c-3p suppressed the Bcl-2 protein levels in $\mathrm{H}_{2} \mathrm{O}_{2}$ exposed $\mathrm{N} 9$ cells (Fig. 4d). The present outcomes suggested that, up-regulation of miR-466c-3p caused a significant decrease in apoptotic cells among the hydrogen peroxide treated N9 cells compared to control, however it was observed that the attenuating effect of miR-466c-3p on N9 cells was blocked significantly with upregulation of Bcl-2 (Fig. 4e, $\mathrm{P}<0.01$ ). Also, over-expression of Bcl-2 halted the effects of miR$466 c-3 p$ blockade activity of caspase-3 in hydrogen peroxide exposed N9 cells (Fig. 4f, $\mathrm{P}<0.01$ ). All together, the findings indicated that miR-466c-3p could suppress apoptosis in neuronal cells via suppressing Bcl-2 in hydrogen peroxide exposed N9 microglia cells.

\section{miR-466c-3p over-expression inhibits the mitochondrial apoptotic pathway}

The pro-apoptotic proteins Bax and Bcl-2 can cause liberation of cytochrome C (Liu et al. 2008; Noguchi et al. 1999). In apoptosis process, overexpression of Bcl-2 can enhance the process by blocking Bax (Klapsinou et al. 2015). Death receptor apoptosis and mitochondrial apoptosis are the two important apoptosis pathways (Su et al. 2016; Lu et al. 2017a, b). Bcl-2 is identified as one of the important molecule responsible for regulating mitochondrial apoptosis pathway which promotes the release of cytochrome $\mathrm{c}$ in the cytoplasm from mitochondria (De Biase et al. 2005). In line to evaluate, whether miR-466c-3p is involved in regulating the mitochondrial apoptosis pathway via down- regulating the expression of apoptosis associated proteins in SCI rats, western blot study was performed for assessing the expression of Bcl-2, Bax, Caspase-3/-9 and Cleaved Caspase-3/-9 in spinal tissues of rats. We evidenced that the levels of Cleaved Caspase $-3 /-9$ and $\mathrm{Bcl}-2$ were significantly increased whereas, expression of Caspase-3/-9 and Bax were suppressed significantly in SCI rats treated with NC inhibitor compared to sham operated rats. However, upregulation of miR-466c-3p caused a significant decrease in levels of cleaved caspase $-3 /-9$ and Bcl-2 also caused a significant increase in levels of Caspase-3/-9 and Bax in SCI rats treated with miR-466c-3p mimics compared to $\mathrm{NC}$ inhibitor rats (Fig. 5, $\mathrm{P}<0.01$ ). These findings suggested that, upregulation of miR-466c-3p may inhibit the mitochondrial apoptotic pathway via suppression of $\mathrm{Bcl}-2$ in rats after SCI.

\section{Discussion}

Spinal cord injury leads to alterations at both molecular as well as at biochemical levels, these changes lead to generation of free radicals, release of inflammatory mediators, death of neurons and axonal plasticity $(\mathrm{Hu}$ et al. 2013). Earlier, some studies have suggested that spinal cord injury may lead to abnormal expression of miRs and these miRs may alter the functional outcomes as well as the pathophysiology (Malhotra et al. 2001). However, studies highlighting the functional significance of some specific miRs are lacking. Here, we constructed an animal model of SCI followed by microarray analysis for expression of miRs at various time points after inducing SCI. We observed that SCI caused deregulation in expression of miRs in rats submitted to SCI among the miRs, miR$466 c-3 p$ was the most significantly down-regulated in the spinal cord tissue. Also, it was found that upregulation of miR-466c-3p attenuated SCI by improving functional recovery, decrease in the size and volume of lesions and also deceased apoptosis. It was also evidenced that, upregulated miR-466c-3p caused inhibition of Bcl-2 in N9 microglia cells via binding to the $3^{\prime} \mathrm{UTR}$ region, this was confirmed when it was tested that upregulated Bcl-2 caused inhibition in the protective effect of miR466c-3p in the N9 cells exposed to hydrogen peroxide. The present work demonstrated the protective role of miR-466c-3p in SCI animal model via blocking the mitochondrial apoptosis cascade.

Literature previously has confirmed that miR-466c-3p is one of the aberrantly expressed $\mathrm{miR}$ in the neurons (Druz et al. 2011). The study showed that miR-466c-3p was one of the most down-regulated miR during the SCI specifically during the 13th and 14th day post-SCI. Parallel to these findings, the present results showed that SCI caused aberrant expression miRs and miR-466c-3p was one of the most significantly suppressed miR in the rats subjected to SCI. The present study also suggested that, up-regulation of miR-466c-3p in rats by treating them with miR-466c-3p mimics improved the motor function as evaluated by BBB scoring; decreased the lesion volume and also suppressed the apoptosis of neuronal cells in $\mathrm{SCI}$ induced rats. These findings demonstrated that miR$466 \mathrm{c}-3 \mathrm{p}$ shows protective role on rats induced to SCI.

Mitochondria play a vital role in the process of apoptosis by releasing important mediators such as cytochrome c (Zou et al. 1999). Bcl-2 is an important apoptosis regulator, it has been identified to increase the permeability of the mitochondrial membrane causing release of cytochrome $\mathrm{c}$ in the cytoplasm (Zou et al. 1999). Cytochrome c is responsible for amplifying the caspase-9 pathway in the mitochondrial apoptosis cascade which in response triggers Caspase-3 to active Caspase-3 (Zou et al. 1999). In the present study, 

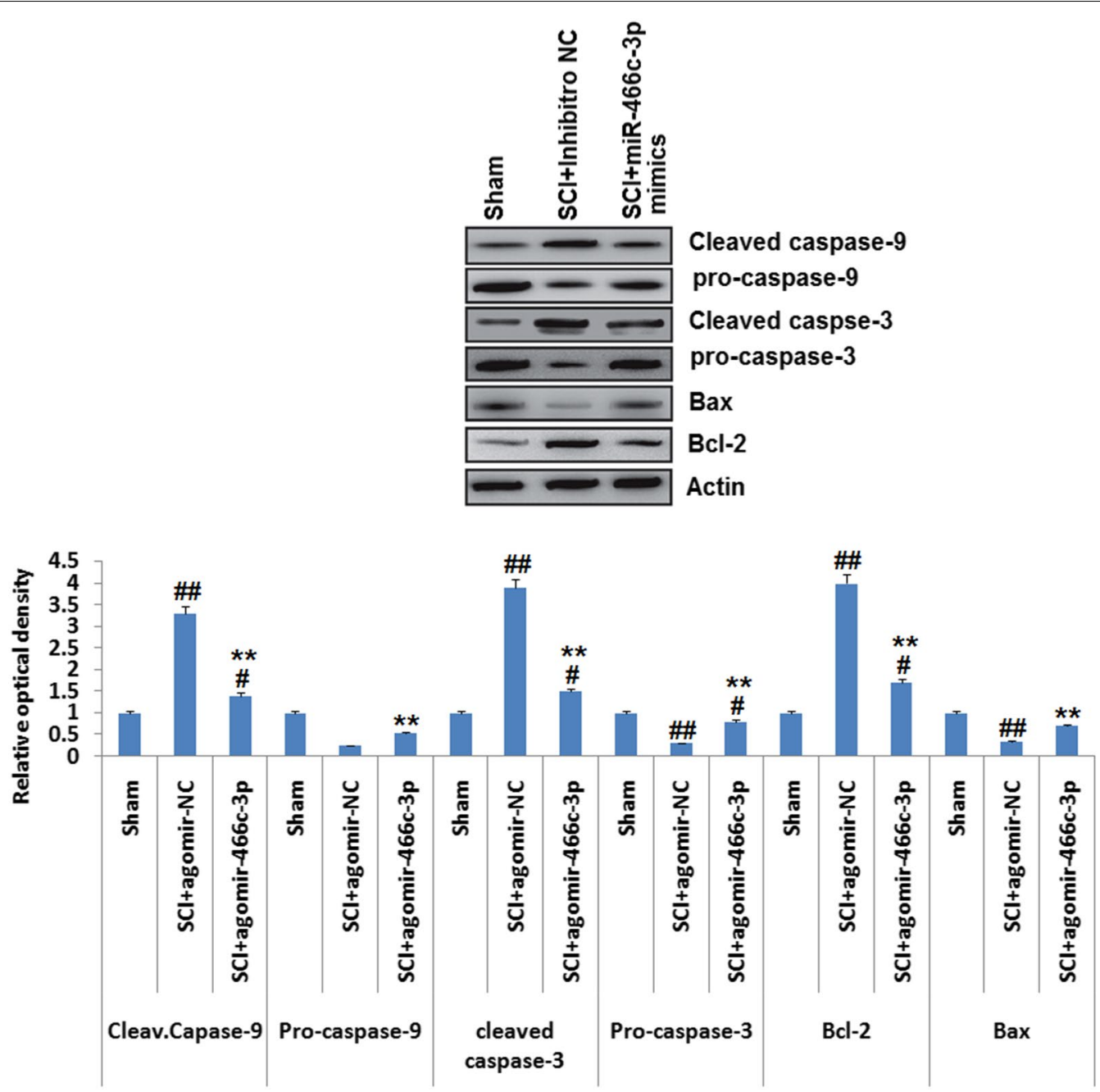

Fig. 5 The experimental animals were induced with spinal cord injury and were exposed to miR-466c-3p mimics or agomir-negative control followed by western blot analysis for determining the expression of cleaved-caspse-9, cleaved-caspase-3, pro-cspase-3, Bcl-2 and Bax in the spinal cord tissue subjected to injury, actin was selected as loading control. The results are mean \pm SD. ${ }^{* *}<0.01$ compared to spinal cord injury rats + treated with agomir-negative control; $\# \mathrm{P}<0.05, \# \# \mathrm{P}<0.01$ compared to sham operated rats

miR-466c-3p suppressed the expression of Bcl-2 via targeting $3^{\prime} \mathrm{UTR}$ in N9 microglia cells. In addition to this, the results of correlation analysis suggested a negative correlation between expression of miR-466c-3p and $\mathrm{Bcl}-2$ in spinal cord tissue of SCI induced rats, indicating $\mathrm{Bcl}-2$ as a potential target of miR-466c-3p in rats. Hence, it may be speculated that miR-466c-3p may modulate the mitochondrial apoptosis cascade via blocking the expression of Bcl-2 in SCI rats. The present findings suggested that over-expression of miR$466 \mathrm{c}-3 \mathrm{p}$ suppressed the expression of $\mathrm{Bcl}-2$, cleaved caspase-3/-9 and enhanced the expression of Bax, Pro-caspase-3/-9 in the spinal cord tissues of SCI rats. Altogether, the results suggested that miR-466c-3p may exhibit protective effect in SCI via inhibiting the mitochondrial apoptosis pathway. However, further studies are needed for evaluating in-depth molecular mechanism for miR-466c-3p in SCI.

The current work showed that SCI causes abnormal expression of $\mathrm{miR}$ in SCI induced rats, among them miR-466c-3p was one of the highly suppressed miRNAs in spinal cords of SCI rats. Also it was noticed that overexpression of miR-466c-3p improved the motor function, decreased lesion size and volume and suppressed apoptosis of neurons in rats subjected to SCI. The present findings also suggested that miR-466c-3p targets Bcl-2 in N9 microglia cells and can extend attenuating effect in SCI via inhibiting the apoptosis cascade in mitochondria, proposing that miR-466c-3p may be a potential and novel therapeutic target in treating SCI. 


\section{Acknowledgements}

We express thanks to the staff and management of the Beijing Jishuitan Hospital, Beijin, China.

\section{Authors' contributions}

$Y A, J L, Q Y$ and MF all were associated planning the work The experiments were carried out by $Y A$, JL,QY and MF whereas QY and YA were also involved in data handling and processing. All the authors contributed equally in preparing the manuscript. All authors read and approved the final manuscript.

\section{Funding}

The work was self financed.

\section{Availability of data and materials}

The supporting data for present findings are under ethics laws of university and is hence not presented here.

\section{Ethics approval and consent to participate}

The experiments received approval from the Beijing Jishuitan Hospital, Beijin China. The animal study protocols were priorly approved by animal ethical review board of Beijing Jishuitan Hospital, Beijin, China.

\section{Consent to publication}

Not applicable.

\section{Competing interests}

The authors declare no competing interests.

Received: 22 March 2020 Accepted: 23 May 2020

Published online: 15 June 2020

\section{References}

Bak M, Silahtaroglu A, Møller M, Christensen M, Rath MF, Skryabin B, Tommerup N, Kauppinen S (2008) MicroRNA expression in the adult mouse central nervous system. RNA 14:432-444

Balaban RS, Nemoto S, Finkel T (2005) Mitochondria, oxidants, and aging. Cell 120:483-495

Bartel DP (2009) MicroRNAs: target recognition and regulatory func-tions. Cell $136: 215-233$

Basso DM, Beattie MS, Bresnahan JC (1995) A sensitive and reliable locomotor rating scale for open field testing in rats. J Neurotrauma 12:1-21

Blight AR (2002) Miracles and molecules-progress in spinal cord repair. Nat Neurosci 5(Suppl 1):S1051-S1054

Chen Y, Cao S, Xu P, Han W, Shan T, Pan J, Lin W, Chen X, Wang X (2016) Changes in the expression of miR-34a and its target genes following spinal cord injury in rats. Med Sci Monit 22:3981-3993

Croce CM (2009) Causes and consequences of microRNA dysregula-tion in cancer. Nat Rev Genet 10:704-714

De Biase A, Knoblach SM, Di Giovanni S, Fan C, Molon A, Hoffman EP, Faden Al (2005) Gene expression profiling of experi-mental traumatic spinal cord injury as a function of distance from impact site and injury severity. Physiol Genomics 22:368-381

Druz A, Chu C, Majors B, Santuary R, Betenbaugh M, Shiloach J (2011) A novel microRNA mmu-miR-466 $\mathrm{h}$ affects apoptosis regulation in mammalian cells. Biotechnol Bioeng 108(7):1651-1661

He J, Xiao Y, Casiano CA, Zhang L (2000) Role of mitochondrial cytochrome C in cocaine-induced apoptosis in coronary artery endothelial cells. J Pharmacol Exp Ther 295:896-903

Hu JZ, Huang JH, Zeng L, Wang G, Cao M, Lu HB (2013) Anti-apoptotic effect of microRNA-21 after contusion spinal cord injury in rats. J Neurotrauma 30:1349-1360

Kawabata H, Setoguchi T, Yone K, Souda M, Yoshida H, Kawahara K, Maruyama I, Komiya S (2010) High mobility group box 1 is upregulated after spinal cord injury and is associated with neuronal cell apoptosis. Spine (Phila Pa 1976) 35:1109-1115
Klapsinou E, Argyri E, Panotopoulou E, Daskalopoulou D, Patsouris E, Nonni A, Lazaris AC, Thomopoulou GH (2015) Bax and Bak expression in cervical smears of women with low- and high-risk HPV types: a study of 120 cases. J Cytol 32:223-229

Kosik KS (2006) The neuronal microRNA system. Nat Rev Neurosci 7(911-920):2006

Li J, Huang C-Y, Zheng RL, Cui KR, Li JF (2000) Hydrogen peroxide induces apoptosis in human hepatoma cells and alters cell redox status. Cell Biol Int 24:9-23

Li P, Nijhawan D, Wang X (2004) Mitochondrial activation of apop-tosis. Cell 116(2 Suppl):S57-S61

Liu H, Qin CK, Han GQ, Xu HW, Ren WH, Qin CY (2008) Synthetic chenodeoxycholic acid derivative, HS-1200, induces apoptosis of human hepatoma cells via a mitochondrial pathway. Cancer Lett 270:242-249

Liu NK, Wang XF, Lu QB, Xu XM (2009) Altered microRNA expression following traumatic spinal cord injury. Exp Neurol 219:424-429

Liu G, Keeler BE, Zhukareva V, Houlé JD (2010) Cycling exercise affects the expression of apoptosis-associated microRNAs after spinal cord injury in rats. Exp Neurol 226:200-206

Lu Z, Chen H, Zheng XM, Chen ML (2017a) Experimental study on the apoptosis of cervical cancer Hela cells induced by juglone through c-Jun $\mathrm{N}$ terminal kinase/c-Jun pathway. Asian Pac J Trop Med 10:572-575

Lu Z, Chen H, Zheng XM, Chen ML (2017b) Experimental study on the apoptosis of cervical cancer Hela cells induced by juglone through c-Jun Nterminal kinase/c-Jun pathway. Asian Pac J Trop Med 10:572-575

Malhotra R, Lin Z, Vincenz C, Brosius FC (2001) III: hypoxia induces apoptosis via two independent pathways in Jurkat cells: differential regulation by glucose. Am J Physiol Cell Physiol 281:C1596-C1603

Nicholson DW, Thornberry NA (1997) Caspases: killer proteases. Trends Biochem Sci 22:299-306

Noguchi K, Kitanaka C, Yamana H, Kokubu A, Mochizuki T, Kuchino Y (1999) Regulation of c-Myc through phosphorylation at Ser-62 and Ser-71 by c-Jun N-terminal kinase. J Biol Chem 274:32580-32587

Rabchevsky AG, Patel SP, Springer JE (2011) Pharmacological interventions for spinal cord injury: where do we stand? How might we step forward? Pharmacol Ther 132:15-29

Reuter S, Eifes S, Dicato M, Aggarwal BB, Diederich M (2008) Modulation of anti-apoptotic and survival pathways by curcumin as a strategy to induce apoptosis in cancer cells. Biochem Pharmacol 76:1340-1351

Rojo Al, McBean G, Cindric M, Egea J, López MG, Rada P, Zarkovic N, Cuadrado A (2014) Redox control of microglial function: molecular mechanisms and functional significance. Antioxid Redox Signal 21(12):1766-1801

Su CC, Lee Kl, Chen MK, Kuo CY, Tang CH, Liu SH (2016) Cantharidin induced oral squamous cell carcinoma cell apoptosis via the JNK-regulated mitochondria and endoplasmic reticulum stress-related signaling pathways. PLOS ONE 11:e0168095

Tang Y, Ling ZM, Fu R, Li YQ, Cheng X, Song FH, Luo HX, Zhou LH (2014) Timespecific microRNA changes during spinal motoneuron degeneration in adult rats following unilateral brachial plexus root avulsion: ipsilateral vs. contralateral changes. BMC Neurosci. 15:92

Thuret S, Moon LD, Gage FH (2006) Therapeutic interventions after spinal cord injury. Nat Rev Neurosci 7:628-643

Wang J, Jiao Y, Cui L, Jiang L (2017) miR-30 functions as an oncomiR in gastric cancer cells through regulation of P53-mediated mito-chondrial apoptotic pathway. Biosci Biotechnol Biochem 81:119-126

Yune TY, Lee JY, Jung GY, Kim SJ, Jiang MH, Kim YC, Oh YJ, Markelonis GJ, Oh TH (2007) Minocycline alleviates death of oligodendrocytes by inhibiting pro-nerve growth factor production in microglia after spinal cord injury. J Neurosci 27:7751-7761

Zou H, Li Y, Liu X, Wang X (1999) An APAF-1.cytochrome c multimeric complex is a functional apoptosome that activates procaspase-9. J Biol Chem 274:11549-11556

\section{Publisher's Note}

Springer Nature remains neutral with regard to jurisdictional claims in published maps and institutional affiliations. 